\title{
Benign coronary perforation during percutaneous transluminal coronary angioplasty
}

\author{
B MEIER \\ From the Centre for Cardiology, University Hospital, Geneva, Switzerland
}

SUMMARY In two patients percutaneous transluminal coronary angioplasty was complicated by coronary perforation. In both cases the complication was managed conservatively.

Two cases of coronary perforation or rupture during percutaneous transluminal coronary angioplasty have been reported. One patient was successfully treated with pericardiocentesis and emergency bypass surgery ${ }^{1}$ and the other died despite pericardiocentesis. ${ }^{2}$ We report two cases of coronary artery perforation during angioplasty that resolved spontaneously.

\section{Patients and methods}

CASE 1

A 58 year old man was admitted for repeat percutaneous transluminal coronary angioplasty five months after successful angioplasty of a stenosis of the right coronary artery. He had been free of symptoms for about two months after his initial treatment. Two weeks before repeat angioplasty a control coronary angiogram showed recurrence of the dilated stenosis (Fig. 1a). The patient was taking a regimen of aspirin $100 \mathrm{mg}$ daily, nitrates, and calcium antagonists. After the intravenous administration of $10000 \mathrm{U}$ heparin and intracoronary administration of $0.1 \mathrm{mg}$ nifedipine and $0.2 \mathrm{mg}$ isosorbide dinitrate a steerable guide wire (Schneider 0.014 inch) could be advanced with difficulty $2 \mathrm{~cm}$ past the stenosis where further advancement became impossible. An injection of contrast medium through the catheter tip already beyond the stenosis failed to opacify the distal vessels but resulted in a linear deposit of contrast outlining the inferior pericardial sac (Fig. 1b). The balloon catheter and guide wire were withdrawn proximal to the stenosis. The patient remained haemodynamically stable and free of pain. The guide wire was advanced again, this time deep into a left ventricular branch. The balloon catheter could be advanced easily and a

Requests for reprints to Dr B Meier, Centre for Cardiology, University Hospital, CH-1211 Geneva 4, Switzerland.

Accepted for publication 11 February 1985 distal injection of contrast outlined the artery containing the guide wire. Several dilatations up to 8 atmospheres were performed with a pronounced reduction of the stenosis evidenced by control angiography, which showed no further extravasation. Remnants of the pericardial deposit of contrast were still visible (Fig. 1c). The effects of the heparin were not reversed, and the patient was discharged two days later after an uneventful recovery. Eight months later, when the patient was seen as an outpatient, he was free of symptoms.

\section{CASE 2}

A 60 year old man was admitted to hospital for unstable angina. Cardiac catheterisation showed a normal left ventricle and two moderate $s^{\prime}$ ?noses of the left anterior descending coronary artery after the junction with a diagonal branch, which was totally occluded but faintly collateralised (Fig. 2a). The left circumflex coronary artery showed a non-significant narrowing, and the right coronary artery was normal. The patient's angina remained unstable requiring treatment in the coronary care unit. Five days later percutaneous transluminal coronary angioplasty was performed. After a pretreatment regimen identical to that in case 1 a Schneider $3.0 \mathrm{~mm}$ balloon catheter was advanced over a guide wire (Schneider 0.014 inch), which had been manoeuvered with difficulty across the total occlusion of the diagonal branch. A distal injection of contrast medium opacified the distal portion of the diagonal branch. Several dilatations up to 6 atmospheres were performed. Distal pressure rose from $35 \mathrm{~mm} \mathrm{Hg}$ to $75 \mathrm{~mm} \mathrm{Hg}$. A control injection showed patency of the formerly occluded segment but also extravasation of contrast medium. The patient complained of a dull chest pain but remained haemodynamically stable. The balloon catheter was withdrawn, and angioplasty of the two stenoses in the left anterior descending coronary artery was successfully performed. A final injection of contrast showed 

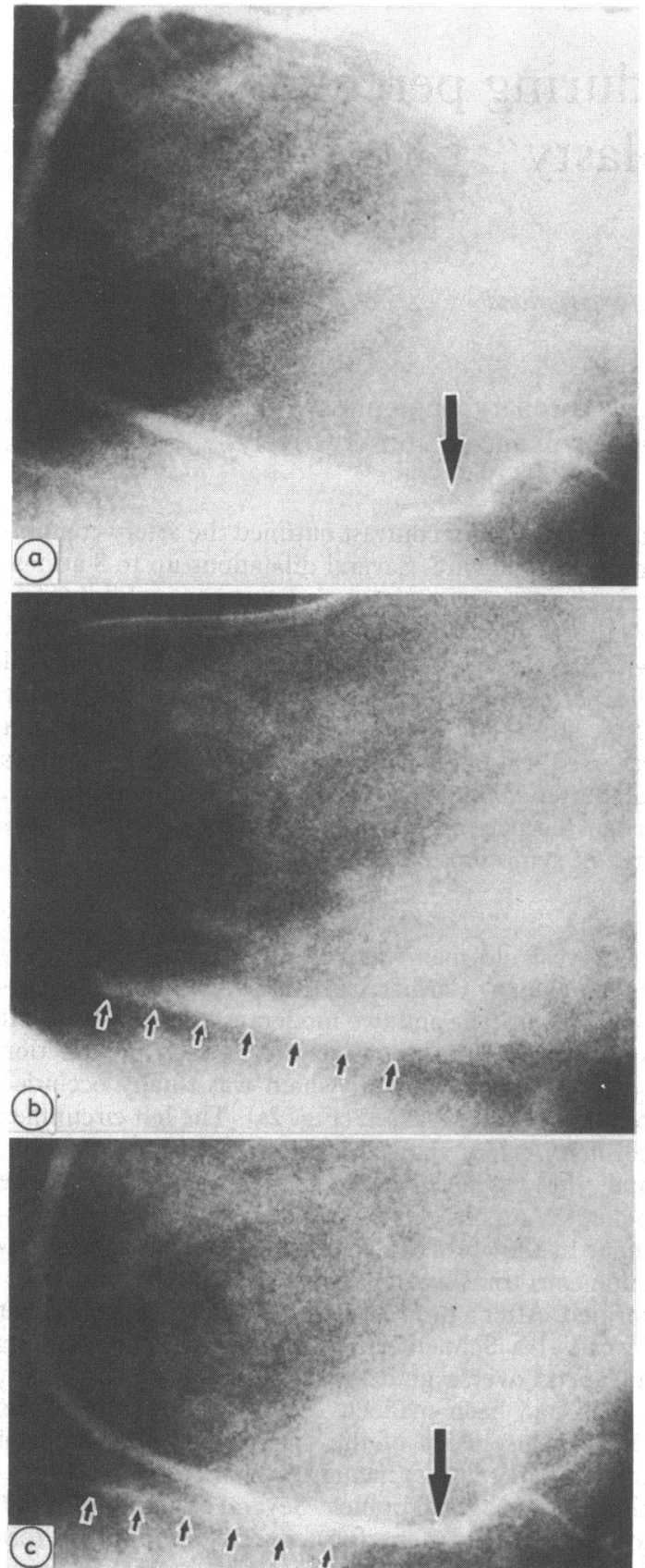

Fig. 1 Case 1: Coronary angiograms showing the right coronary artery in the left anterior oblique projection (a) before angioplasty (stenosis indicated by large arrow); (b) after perforation (contrast deposit is visible in the pericardial sac (small arrows); and (c) after angioplasty (dilated segment indicated by large arrow, remnants of pericardial contrast deposit by small arrows).

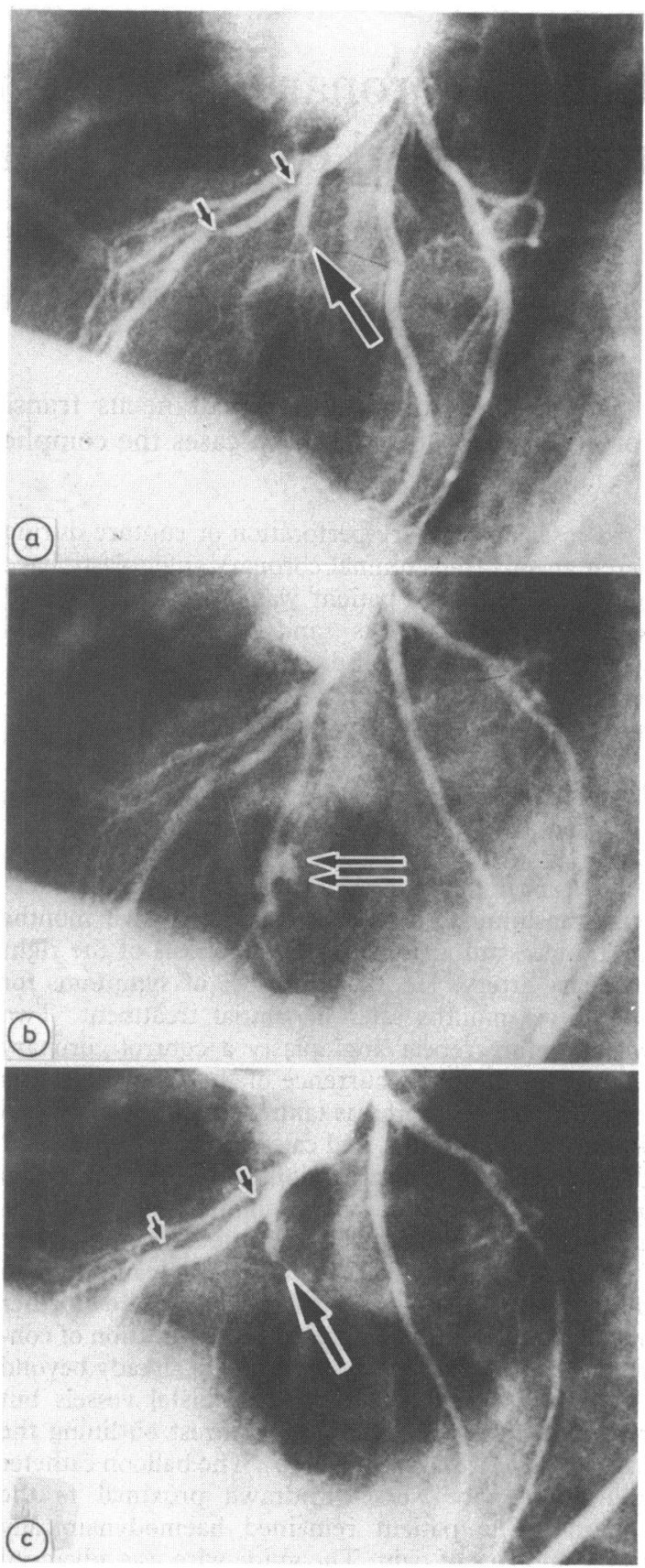

Fig. 2 Case 2: Coronary angiograms showing the left coronary artery in the left anterior oblique projection with cranial angulation (a) before angioplasty (stenosis of left anterior descending coronary artery indicated by small arrows, occlusion of diagonal branch indicated by large arrow), (b) after angioplasty (contrast extravasation indicated by double arrow), and (c) two weeks later (site of previous stenoses of left anterior descending coronary artery indicated by small arrows, reocclusion of diagonal branch indicated by large arrow). 
continuing extravasation of contrast medium at the site of the previous occlusion of the diagonal branch and patency of the vessel itself (Fig. 2b). The contrast deposit around the dilated vessel drained slowly into the pericardium. The effects of heparin were reversed with protamine sulphate, and an intravenous glyceryl trinitrate infusion was continued. Chest pain persisted for about two hours. No haemodynamic alteration, pericardial rub, increase in creatine phosphokinase activity, or electrocardiographic changes were observed. A few days later the patient complained again about exertional angina. Control coronary angiography two weeks after angioplasty showed reocclusion of the diagonal branch at the initial site. The left anterior descending coronary artery was widely patent (Fig. 2c). Repeat angioplasty of the occluded diagonal branch was not attempted, and conservative treatment was recommended. Eight months later the patient was free of symptoms with a regimen consisting of a calcium blocker, nitrates, and aspirin.

\section{Discussion}

Our first case represents perforation of a distal coronary artery with the guide wire and the tip of the balloon catheter. Recognition of the false passage before balloon dilatation prevented enlargement of the perforation and allowed spontaneous sealing. This case emphasises the importance of a distal injection of contrast before balloon filling to determine the correct intraluminal position of the balloon catheter.

Our second case represents a rupture of a relatively small branch as a result of the dilatation process itself.
It is conceivable that both guide wire and balloon catheter were advanced subintimally through the occlusion re-entering the correct lumen distal to it. The correct intraluminal position of the balloon tip before balloon filling was established both by pressure measurement and dye injection. Dilatation with the relatively large balloon resulted in transmural dissection of the arterial wall. Rapid termination of the angioplasty procedure with prompt reversal of heparinisation was probably crucial in preventing cardiac tamponade. Unfortunately, not only the perforation but also the re-established lumen closed spontaneously. The patient, however, still benefited from the successful angioplasty of the strategically more important lesions in the left anterior descending coronary artery.

Drastic measures such as reocclusion of the bleeding vessel with the balloon, pericardiocentesis, or emergency operation suggested or performed in previously reported cases of coronary perforation ${ }^{12}$ were not necessary in these patients. Thus coronary perforation due to balloon angioplasty may resolve spontaneously but still demands careful supervision and readiness for active treatment.

\section{References}

1 Kimbiris D, Iskandrain AS, Goel I, et al. Transluminal coronary angioplasty complicated by coronary artery perforation. Cathet Cardiovasc Diagn 1982; 8: 481-7.

2 Saffitz JE, Rose TE, Oaks JB, Roberts WC. Coronary arterial rupture during coronary angioplasty. $\mathrm{Am} \mathcal{F} \mathrm{Car}$ diol 1983; 51: 902-4. 